\title{
Dynamic Ocean Management for Salmon: Integrating Spatially-explicit Environmental and Fishery Datasets to Describe and Predict Fish Distributions
}

\author{
Jordan T. Watson ${ }^{1}$, Rob Ames², Camille Kohler ${ }^{3}$, Robert Nigh ${ }^{3}$, Robert Ryznar ${ }^{2}$, and Jenny Suter ${ }^{2}$ \\ ${ }^{I}$ Alaska Fisheries Science Center, NMFS, NOAA, 17109 Pt. Lena Loop Rd., Juneau, AK, USA \\ ${ }^{2}$ Pacific States Marine Fisheries Commission, 205 E Spokane St. \#100, Portland, OR 97202, USA \\ ${ }^{3}$ Nexus Data Solutions, 721 W 1st Ave STE. 100, Anchorage, AK 99501, USA
}

Keywords: dynamic ocean management, near real-time, remote sensing, ocean distribution

Dynamic ocean management seeks to use near real-time information to allow for spatially and/or temporally flexible management measures (Maxwell et al. 2015; Hazen et al. 2018; Welch et al. 2019). Recent advances in satellite technology have facilitated such pursuits by making fully automated and quality-controlled datasets both available and readily implemented into a suite of programming environments. Thus, models that are dependent upon environmental information can be updated with as little as several hours of lag-time, allowing managers or enforcement officials to respond in near real-time.

Dynamic models in general can address a suite of different management goals but several unifying principles data acquisition, prediction, dissemination, and automation — guide their development whether they are designed to inform hurricane evacuations, bycatch avoidance, or fish species targeting (Welch et al. 2019). For the purposes of understanding salmon distributions in the high seas, the data acquisition step would be required from two sources. First, acquisition of historical fishing data with locations and dates of non-zero catches and zero catches are necessary to train models. Second, environmental data must be matched to the fishing data; these data can originate either from data collected at the time of fishing or data extracted from satellite data based on dates and locations. Predictions can be trained using the historic catch information and developed via a variety of statistical approaches like gradient boosting, random forests, or other (often machine learning) techniques (e.g., Scales et al. 2017; Hazen et al. 2018; Cimino et al. 2019). Dissemination dictates that in order for dynamic management to be useful, there must be a developed platform such that data predictions can be communicated effectively to the managers, enforcement officials, or other users for whom the approaches were developed. Finally, each of these steps must be updated regularly so that users can respond in near real-time. In the case of high seas salmon fishing, for example, environmental data would need to be updated daily so that enforcement officials could evaluate a need for enforcement action based on updated predictions on movement of fish populations.

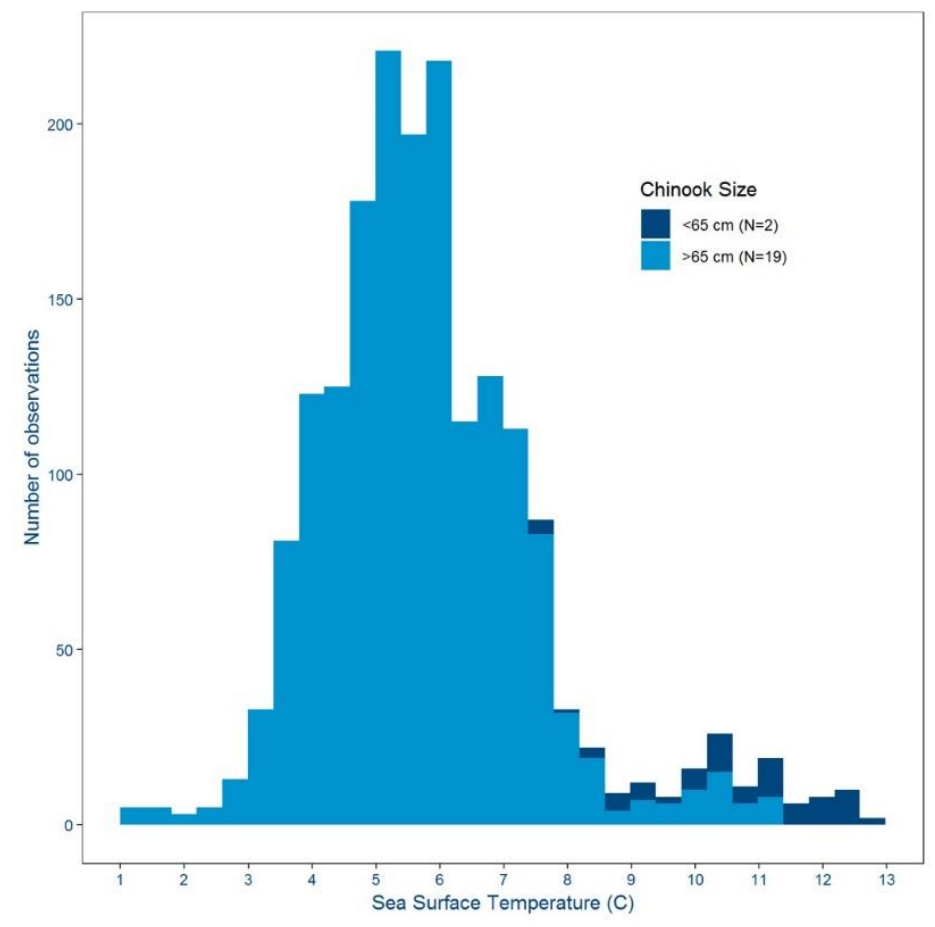

Fig. 1. Distribution of satellite-derived sea surface temperatures for Chinook tag locations.

Distributions of data from Chinook with fork length $<65 \mathrm{~cm}(N=2)$ and Chinook with fork length $>65$ $\mathrm{cm}(N=19)$ are shaded in darker and lighter colors, respectively. 
Our efforts at dynamic ocean management are still in their nascent stages but we have developed two pilot projects that provide proof of concept for each of the four steps described above (acquisition, prediction, dissemination, and automation). The first has explored the acquisition of satellite information on sea surface temperature (SST) for a time series, aggregated into statistical management areas (Watson 2019). These data have been subsequently integrated into a management database at the Alaska Fisheries Science Center that is accessible to a suite of researchers and managers. Furthermore, an additional acquisition algorithm runs each day, updating the data in the database, and thus providing a data acquisition that is automated and disseminated to users daily.

The second pilot project involves acquisition of data on fish distributions and the development of environmentally-explicit predictions. For the initial study, data on the locations of Chinook salmon were provided from satellite tag data (Seitz et al. 2019). Environmental data (SST) for each of the tag records were obtained from a NOAA ERDDAP server (Watson 2019), which yielded distributions for each environmental covariate (Fig. 1). In this case, models were demonstrated with only a single environmental covariate, but future work will include a suite of potential other covariates (e.g., Hazen et al. 2018; Cimino et al. 2019; Watson et al. 2018). This example only utilized a single environmental covariate, SST, so the model for the predictive step was a simple univariate generalized additive model. However, in future efforts, we will explore more adaptive recursive portioning and machine learning approaches. Once the predictive model was trained with Chinook data, we acquired SST data from across the North Pacific Ocean (using the ERDDAP server as described above) (Fig. 2), and we applied these data to the model to predict where expected Chinook abundance was highest (Fig. 3). In the figure, the black lines represent the exclusive economic zone boundaries for each country, facilitating a simple illustration of which high seas areas may be mostly likely to contain salmon for the date of prediction.

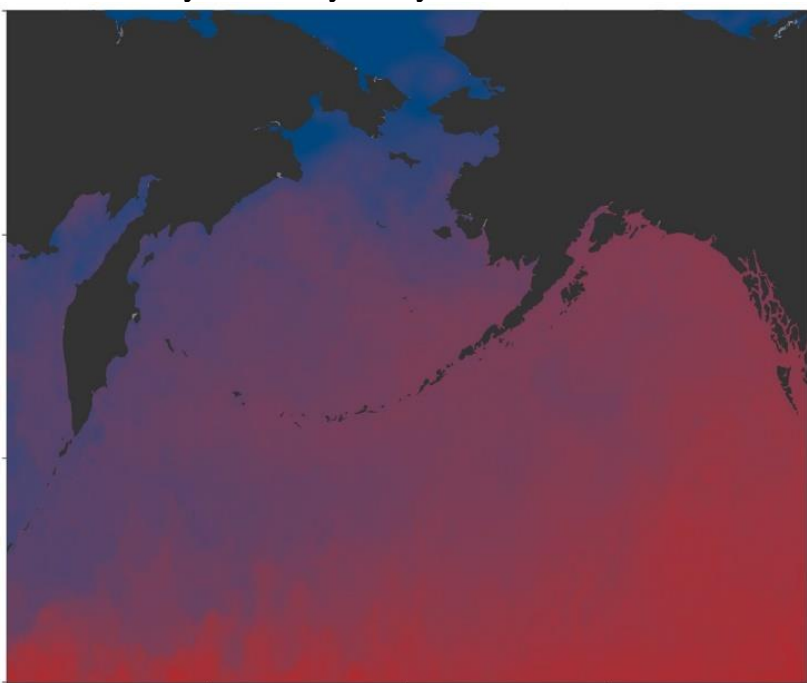

Fig. 2. Example of sea surface temperatures across the North Pacific Ocean acquired from satellite data for 5 May 2019. Redder colors represent warmer temperatures.

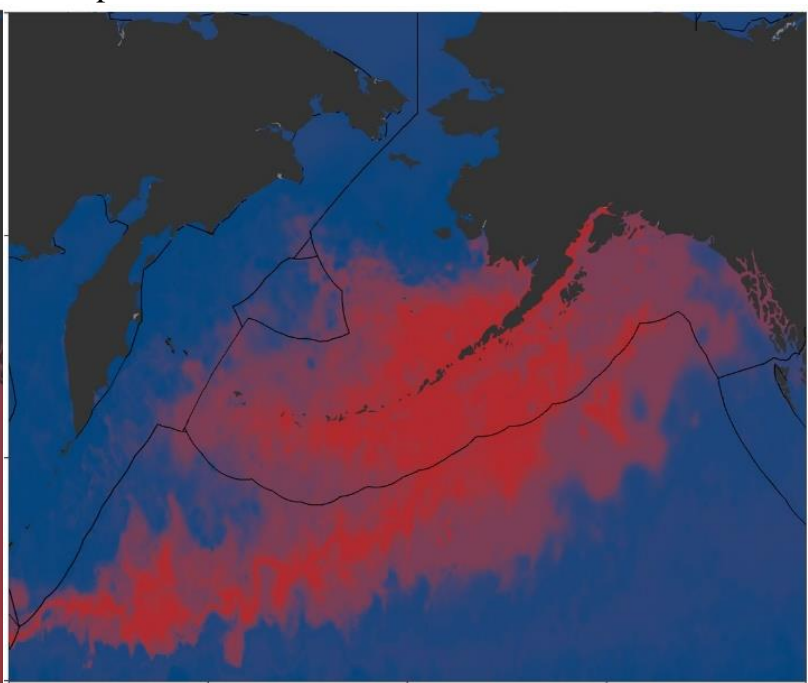

Fig. 3. Example of predicted Chinook salmon distribution using sea surface temperatures from 5 May 2019. Redder colors represent a greater likelihood of salmon occurrence. Black lines overlain are the exclusive economic zone boundaries.

While the work presented here represents only nascent steps towards predicting salmon distributions in the ocean, we illustrate a framework and proof of concept for future operationalization and development of a dynamic ocean management tool. Next steps for this work include compilation of historic salmon catches and fishing efforts that are necessary to train models on the distribution of multiple species of interest. Furthermore, in order for these models to be maximally effective for combating illegal, unreported, and unregulated fishing in the high seas, the dissemination and automation steps will need to integrate fishing vessel location data and must be portable to enforcement infrastructure.

\section{REFERENCES}

Cimino, M.A., M. Anderson, T. Schramek, S. Merrifield, and E.J. Terrill. 2019. Towards a Fishing Pressure Prediction System for a Western Pacific EEZ. Sci. Rep. 9: 461. doi:10.1038/s41598-018-36915-x.

Hazen E.L., K.L. Scales, S.M. Maxwell, D.K. Briscoe, H. Welch, S.J. Bograd, and H. Bailey. 2018. A dynamic ocean management tool to reduce bycatch and support sustainable fisheries. Sci. Adv. 4: eaar3001. 
Maxwell, S. M., E.L. Hazen, R.L. Lewison, D.C. Dunn, H. Bailey, S.J. Bograd, and L.B. Crowder. 2015. Dynamic ocean management: Defining and conceptualizing real-time management of the ocean. Mar. Pol. 58: 42-50.

Scales, K.L., E.L. Hazen, S.M. Maxwell, H. Dewar, S. Kohin, M.G. Jacox, and S.J. Bograd. 2017. Fit to predict? Eco-informatics for predicting the catchability of a pelagic fish in near real time. Ecol. Appl. 27(8): 23132329.

Seitz, A.C., M.B. Courtney, M.D. Evans, and K. Manishin. 2019. Pop-up satellite archival tags reveal evidence of intense predation on large immature Chinook salmon in the North Pacific Ocean. Can. J. Fish. Aquat. Sci. 76(9): 1608-1615. doi:10.1139/cjfas-2018-0490.

Watson J.R., E.C. Fuller, F.S. Castruccio, and J.F. Samhouri. 2018. Fishermen Follow Fine-Scale Physical Ocean Features for Finance. Front. Mar. Sci. 5: 46.

Watson J.T. 2019. Spatial and temporal visualizations of satellite-derived sea surface temperatures for Alaska fishery management areas. Pac. States E-J. Sci. Vis. doi: 10.28966/PSESV.2019.003.

Welch H., E.L. Hazen, S.J. Bograd, M.G. Jacox, S. Brodie, D. Robinson, and K.L. Scales. 2019. Practical considerations for operationalizing dynamic management tools. J. Appl. Ecol. 56: 459-469. 